$12 \%$ ). For traceable partners (73\% of men's; $88 \%$ of women's), 9/157 men's partners and 16/114 women's partners required provider-led PN. Almost all were casual or ex-partners (8/9 men's; 13/16 women's). NNTITs were lowest for ex-partners: 0.6 and 0.45 for men and women, respectively, followed by casual partners: 0.8 and 0.6 , then regular partners: 1.80 and 0.9 .

Discussion Provider-led PN is required more often with casual and ex-partners than with regular partners. This may have greater cost implications for services, but, as casual and ex-partners are likely to have greater numbers of partners themselves, the potential for preventing onward transmission is greater, yielding greater public health benefit. Services should collect data from their index patients on the number and type(s) of partners, as well as the PN method(s) required (patient vs provider referral). Together with cost data, these data were key for demonstrating the cost-benefit of provider-led PN.

\section{P39 THE ACCEPTABILITY AND USE OF SMS TEXT MESSAGING FOR PROVIDER REFERRAL PARTNER NOTIFICATION (PRPN)}

doi:10.1136/sextrans-2012-050601c.39

V Gilbart, ${ }^{*}$ K Town, C M Lowndes. The Health Protection Agency

Background Partner notification is important for STI control. With developments in technology notifying partners is now easier. The HPA was keen to understand the acceptability and use of SMS texting for PRPN for STIs.

Methods A questionnaire distributed to GUM colleagues, cascaded by the Society of Sexual Health Advisers and posted on their website.

Results 65 sites responded. Most (61,93\%) use telephone for 1st attempt for PRPN with texting as preferred second choice $(19,29 \%)$. For 2nd attempt, telephone remained preferred method (51, 79\%) but texting increased with $32(49.2 \%)$ choosing this method; letter use also increased. Majority of sites used texting for PRPN (56, $(86 \%)$; frequency varied-over half using it up to $50 \%$ of the time. $29(45 \%)$ had texting guidelines and 31 (55\%) used texting templates. Messages varied; 33 (59\%) requesting partner make contact, 9 (16\%) naming the infection. Six (11\%) had contact with Information Governance Board about texting; no negative outcomes reported. Two partners complained, not about receiving a text but about being denied the identity of the index patient. 9 (14\%) sites never used texting.

Conclusion Telephone contact was the preferred option for 1st and 2nd attempt for PRPN. Texting was the 2 nd most used method and was seen as acceptable within the clinic and also with Information Governance Board. Frequency varied considerably and message content differed but even if used infrequently, was seen as important if other methods failed. Reassuringly no complaints about texting were received from partners. A small number of sites never use texting.

\section{P40 LOSING THE BATTLE? TIME TO FOCUS THE RISK DISCUSSION WITH HIV-POSITIVE MSM}

doi:10.1136/sextrans-2012-050601c.40

S Perera, ${ }^{*}$ S McCormack, N Nwokolo. Chelsea and Westminster Hospital NHS Foundation Trust, London, UK

Background BHIVA 2008 Guidelines advocate development of prevention messages promoting sexual health of HIV-positive individuals to minimise onward transmission and acquisition of infections prevalent in such individuals, for example HCV and LGV.
Objectives To assess the extent of unprotected anal intercourse (UAI) in an HIV-positive population attending a London sexual health clinic. To assess transmission and acquisition risks. To describe the nature of sexual health discussions with health care providers.

Methods 50 randomly selected notes of HIV-positive patients were reviewed. Data were collected on demographics, ART, viral load (VL), sexual partners and topics discussed.

Results 66\% were from the UK and 74\% were $>30.47$ (94\%) were MSM/Bisexual; 2 (4\%) heterosexual females; for 1 (2\%) male IVDU, orientation, partners and discussion were not documented. In 8/47 MSM it was unclear if AI had occurred; 1/47 reported AI but condom use was not documented. In 38 sufficiently complete records, all reported AI: 7 (18\%) protected AI only, 12 (32\%) UAI with positive partners only; $19(50 \%)$ reported UAI with positive and negative partners, although $9 / 19$ (47\%) had VL $<500.7 / 10$ MSM reporting UAI who had VL $>500$ used recreational drugs although only one reported doing so during sex. Discussion was not documented in 17 (34\%) notes, and in 12 focus was only on transmission. 19 topics were discussed with no consistency but could be classified as transmission or acquisition related. Transmission risks were discussed in 15 (79\%) of the 19 reporting UAI with negative partners; acquisition risks were discussed in 17 (55\%) of 31 at risk.

Conclusions Although UAI was reported by the majority of MSM only 10 reported a clinically relevant risk of transmitting HIV, but the majority were at risk of acquiring HCV/LGV. The diversity in topics noted as discussed suggests that simpler, more focused messages of risk reduction would be helpful to sexual health service users and providers.

\section{P41 WOMEN'S HEALTH STUDY OF MYCOPLASMA GENITALIUM: A FEASIBILITY STUDY}

doi:10.1136/sextrans-2012-050601c.41

${ }^{1} \mathrm{~S}$ Dave, ${ }^{*} \mathrm{~S}$ R Kerry, ${ }^{2} \mathrm{P}$ Oakeshott, ${ }^{3} \mathrm{~F}$ McGregor, ${ }^{3} \mathrm{E}$ Cannon, ${ }^{1} \mathrm{~J} \mathrm{M}$ Stephenson. ${ }^{1}$ University College London, London, UK; ${ }^{2} S$ t. George's University of London, London, UK; ${ }^{3}$ CNWL Camden Provider Services, London, UK

Background The role of Mycoplasma genitalium ( $~ 3 \%$ prevalence in women) in PID is unclear. An opportunity exists to study its natural history since there are no UK treatment guidelines. We conducted a feasibility study.

Objectives In women attending GUM clinics to determine: (1) recruitment and follow-up rates after 3 and 6 months, (2) feasibility of obtaining NHS numbers, (3) facilitators and barriers to follow-up. Methods Women aged 16-24 years recruited from two GUM clinics completed a questionnaire and provided two self-taken vaginal samples. One sample was tested for chlamydia. We are testing for $M$ genitalium and conducting 3-month follow-up.

Results Recruitment rate was 67\% (199/296). Non-responders were similar in age to responders (20.6 SD 2.5 vs 20.7 SD 2.5 years) but more likely to come from ethnic minority groups: $56 \%(37 / 66)$ vs $36 \%(71 / 195), p<0.05$. Among responders, mean sexual debut age was 16.3 years (range $12-23 n=198) ; 59 \%(116 / 195)$ reported $\geq 2$ sexual partners and $17 \%(31 / 186)$ consistently used condoms in the preceding 12 months. $8.2 \%(16 / 197)$ had chlamydia and $0.5 \%$ (1/ 197) gonorrhoea on testing. At recruitment 196 participants consented to NHS numbers being obtained and used to access medical records; 197 participants agreed to 3-month follow-up. We have been able to contact $\sim 50 \%$ of participants for follow-up.

Discussion Most women consented to NHS number and medical record access. This is important for investigating long-term sequelae of STIs. Fewer women took part in follow-up than agreed at recruitment. It is important to understand the reasons for this. 